\title{
Multi-scale Computational Models of Pro-angiogenic Treatments in Peripheral Arterial Disease
}

\author{
Feilim Mac Gabhann, James W. Ji, and Aleksander S. Popel \\ Department of Biomedical Engineering, Johns Hopkins University School of Medicine, 720 Rutland Avenue, Baltimore, MD
} 21205, USA

(Received 24 September 2006; accepted 5 March 2007; published online 10 April 2007)

\begin{abstract}
The induction of angiogenesis is a promising therapeutic strategy for the amelioration of peripheral arterial disease (PAD). This occlusive disease results in muscle ischemia, and neovascularization is a route to increasing the perfusion in the tissue. The vascular endothelial growth factor (VEGF) family of potent pro-angiogenic cytokines is a potential therapeutic agent, increasing VEGFreceptor signaling on tissue vasculature. To investigate the effects of possible therapies on the VEGF concentrations and gradients within the tissue, we consider three such strategies: VEGF gene therapy (e.g. by adeno-associated virus); VEGF cell-based therapy (injected myoblasts that overexpress VEGF); and chronic exercise (which upregulates VEGF receptor expression). The multi-scale computational model used to investigate these strategies is an integration of several components: an anatomical description of the muscle geometry and cell types; microvascular blood flow; tissue oxygen distribution; VEGF secretion from muscle fibers; VEGF transport through interstitial space; and VEGF-receptor binding on microvascular endothelial cells. Exercise training, which results in increased VEGF secretion in hypoxic tissue and increased VEGF receptor expression, exhibits increases in both VEGF concentration and VEGF gradients, and is predicted to be more effective than the other, VEGF-only treatments.
\end{abstract}

Keywords-Vascular endothelial growth factor (VEGF), Angiogenesis, Human therapy, Mathematical model, Muscle, Endothelial cell, Theoretical model.

\section{INTRODUCTION}

Angiogenesis (or neovascularization), the growth of new blood vessels from existing microvasculature, is implicated as a pathological factor in many diseases, including cancer, ${ }^{11,12}$ diabetic retinopathy, ${ }^{28}$ age-related

Address correspondence to Feilim Mac Gabhann, Department of Biomedical Engineering, Johns Hopkins University School of Medicine, 720 Rutland Avenue, Baltimore, MD 21205, USA. Electronic mail: feilim@jhu.edu

Feilim Mac Gabhann and James W. Ji contributed equally to this work. macular degeneration ${ }^{54}$ and arthritis. ${ }^{37}$ However, in contrast to these diseases of hypervascularization, there are also pathologies for which increased angiogenesis is being pursued as a potential treatment. These include myocardial ischemia, ${ }^{1,21}$ delayed wound healing ${ }^{60}$ and peripheral obstructive vascular diseases, in particular peripheral arterial diseases (PAD). ${ }^{21,22,49}$

PAD patients experience insufficient blood flow during exercise (intermittent claudication) or rest. PAD affects $15 \%$ of adults over 55 ; patients have a $25 \%$ higher mortality rate, and amputation is often performed (50,000 cases/year in the United States). ${ }^{10,65}$ Exercise training is currently regarded as the most effective treatment for intermittent claudication. However, its therapeutic mechanisms are poorly understood and it is applied only to select patients. ${ }^{50}$

Vascular endothelial growth factor (VEGF) is a family of pro-angiogenic cytokines. The proteins of this family are key promoters of both physiological and pathological angiogenesis. In in vitro studies, they increase survival, proliferation and migration of endothelial cells; $;{ }^{55}$ in vivo, they act as a chemoattractant and direct capillary growth. ${ }^{15,20,52}$ In this study, we use the term VEGF to refer to the two most common protein products of the rat VEGF-A gene, $\mathrm{VEGF}_{120}$, and $\mathrm{VEGF}_{164}\left(\mathrm{VEGF}_{121}\right.$ and $\mathrm{VEGF}_{165}$, respectively, in humans). The difference between these two proteins is the ability of the longer isoform to bind to both glycosaminoglycans (GAGs) in the interstitial space and Neuropilin co-receptors on endothelial cells. ${ }^{57,66}$

Due to the dual effects of VEGF as mitogen and chemoattractant, the angiogenic response to VEGF depends on both: VEGF concentration (and the resulting formation of VEGF-VEGF receptor complexes on endothelial cells); and the formation of extracellular gradients of VEGF concentration. These are the two primary quantities that we report in this computational study. 
Currently, experimental measurements at the microvascular scale of VEGF concentrations and concentration gradients are not possible in vivo. While this makes it difficult to compare the results of these simulations to experimental data, it is the ability of this model to predict these values that makes it novel and uniquely useful in furthering the understanding of the behavior of the VEGF system at the single-vessel, and single-cell, level.

The models on which these simulations are based have been developed over several previous studies. $^{24,25,31,32,34,36}$ The components of these models include: an anatomical description of the muscle geometry and cell types, including muscle fibers, blood vessels, extracellular matrix $(\mathrm{ECM})$ and basement membranes (BMs); blood flow in the microvasculature; oxygen distribution in the tissue; oxygen-dependent VEGF secretion from the muscle fibers; VEGF transport through the interstitial space, including sequestration by glycosaminoglycans of the ECM and BMs; and VEGF-receptor binding on endothelial cells of the microvasculature.

The models are coupled at several points. Anatomy (geometry) is a common basis for the blood flow, oxygen transport, and VEGF transport models. The results of the blood flow model are an input into the oxygen transport model. The oxygen concentrations that are predicted are integrated across the muscle fibers and used to calculate the local VEGF secretion rate, a key driver of the VEGF transport model. The oxygen-VEGF secretion relationship ${ }^{24,32}$ is based on experimental data on the regulation by oxygen of the expression of VEGF via its transcription factor, hypoxia-inducible factor $1 \alpha(\mathrm{HIF} 1 \alpha) .{ }^{26,29,48,59}$

The complexity of this system-including the interactions of VEGF ligands with their receptors and co-receptors, as well as the development of spatial gradients in an anatomically representative geometry-makes it impossible to achieve a quantitative understanding using experimental methods alone. We build here on our previously constructed models: studying VEGF interactions with VEGF receptors in healthy and ligated rat extensor digitorum longus $(\mathrm{EDL})^{24,31,32}$ and in human vastus lateralis muscle, ${ }^{36}$ and interactions with Neuropilin-1 (NRP1) and placental growth factor (PlGF) in vitro. ${ }^{33,34}$

The most common animal model of PAD is surgical ligation of the femoral or iliac arteries. . $^{3,29,44,47,51,53}$ Although this does not completely reproduce the symptoms of PAD, it has become a valuable tool and a useful correlation. Thus, the simulations we present here are for rat EDL muscle following femoral ligation. The muscle loses perfusion due to the loss of a major artery, but re-perfusion begins within the first 2 weeks as a result of collateralization (i.e. the dilation or remodeling of smaller arteries and microvessels). ${ }^{8,18}$ At rest, the recovery in tissue oxygen content is sufficient, but limb activity is hindered as exercise exerts demands on the provision of oxygen to the tissue. . $^{3,17,44}$ Our models of VEGF transport in EDL muscle have been applied to resting muscle, ${ }^{31}$ exercising muscle, ${ }^{32}$ and a femoral ligation model of PAD in resting and exercising muscle. $^{24}$

Here, we apply the model to the prediction of VEGF concentration and VEGF gradient formation following three post-ligation therapeutic interventions in EDL muscle: VEGF gene therapy, using an adenoassociated virus (AAV)-type construct; VEGF cellbased therapy, using VEGF-overexpressing myoblasts that incorporate into muscle tissue following injection; and chronic exercise, which increases VEGF receptor expression, and therefore increases the signaling potential of VEGF.

These simulations will aid in understanding of the role of VEGF, VEGF receptors, and VEGF gradients in PAD, either through physiological changes (e.g. as a result of exercise) or through protein, gene or cellularbased therapies. The model is also general enough to be applicable to other tissue types in the future.

\section{METHODS}

The model used here was developed over several studies and all aspects are described in detail there. $^{24,25,31,32}$ Here we present an overview of the structure of the model, and some of the important aspects of physiology and molecular, and cellular biology included. We present the reaction-diffusion equations governing VEGF transport in a general form, not reported previously. The full set of equations is provided in the online supplement.

\section{Anatomy}

The three-dimensional model system is a $400 \times 208 \times 800 \mu \mathrm{m}^{3}$ volume of rat EDL muscle, including muscle fibers, microvessels, ECM and BMs. The fiber diameters, vessel diameters, capillary density, volume of the interstitial space, and thickness of BMs are based on experimental observations both in rest and exercise (Fig. 1). $3,9,14,20,27,30,38-40,42,59,62$ This threedimensional model is used in calculations of blood flow and oxygen distributions. However, due to the complexity of the system and the number of the parameters that are of physiological interest, we choose a twodimensional cross section of the tissue $\left(400 \times 208 \mu \mathrm{m}^{3}\right.$, boxed in Fig. 1a) for the VEGF transport calculations. We have shown previously that VEGF gradients in the longitudinal direction (parallel to the muscle fibers) are an order of magnitude smaller, on average, than the 

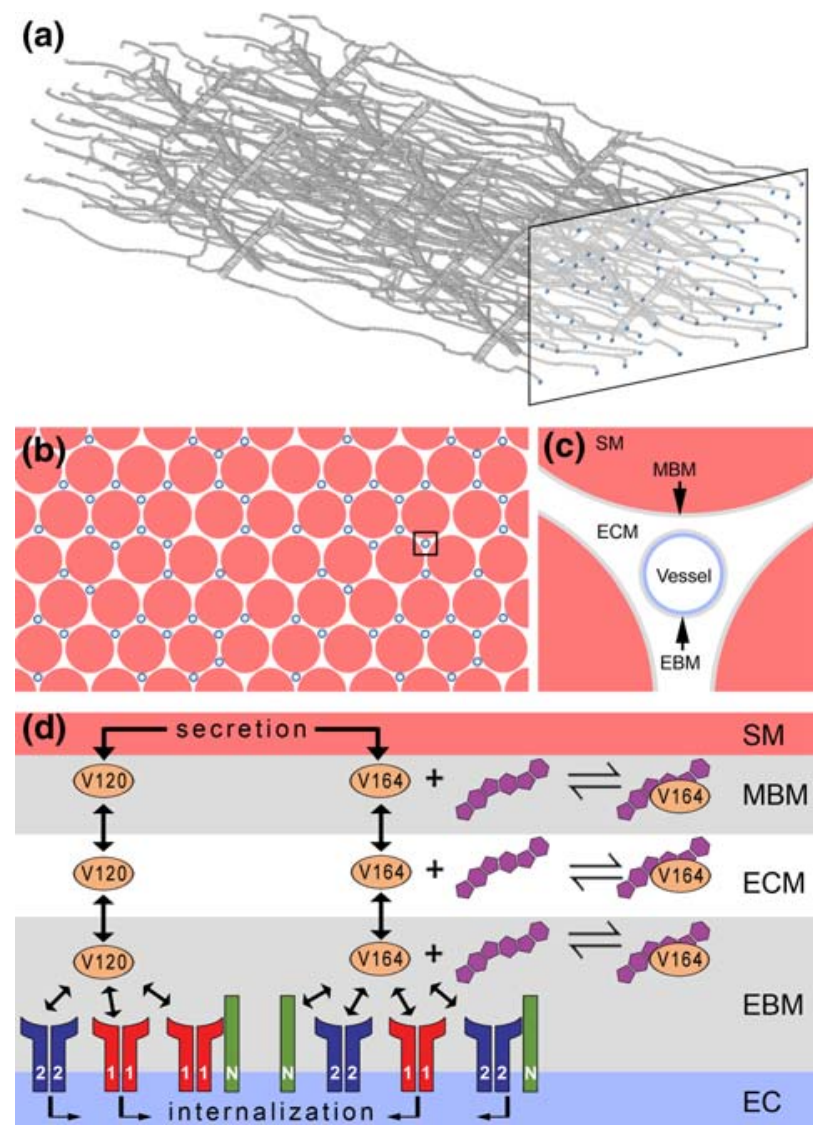

FIGURE 1. Schematics of muscle model geometry and VEGF transport. (a) Three-dimensional microvascular network of simulated rat EDL muscle. The muscle fibers, parallel to the capillaries, have been removed for illustration purposes. Paired arterioles and venules (larger vessels) act as the inlet and outlets for the capillaries. Blue discs denote where capillaries intersect a cross section (boxed) of the tissue. (b) Cross section of rat EDL muscle (boxed area in (a)). Skeletal Muscle (SM) fibers (red) and capillaries (blue) are separated by interstitial space (white). SM fibers are regularly spaced and hexagonally packed. (c) Detail of the interstitial space near a capillary (boxed area in (b)). Muscle fibers are surrounded by a myocyte basement membrane (MBM); capillaries (formed by endothelial cells) are surrounded by an endothelial basement membrane (EBM). The ECM lies between the EBM and MBM. VEGF diffuses throughout the interstitial space: ECM, EBM, and MBM. (d) Diffusion of VEGF isoforms and binding to receptors. $\mathrm{VEGF}_{120}$ and $\mathrm{VEGF}_{164}$ are secreted from SM into MBM and diffuse throughout the interstitial space. Only VEGF 164 binds to glycosaminoglycans (GAGs), which exist at different concentrations in each layer. Near the endothelial cell surface (in the EBM), VEGF can interact with VEGFR1 (marked 1), VEGFR2 (2) and NRP1 (N). VEGF $_{164}$ can bind both VEGFR2 and NRP1 simultaneously, binding first one and then the other. VEGFR1 and NRP1 interact directly, and this complex binds VEGF $_{120}$ but not VEGF $_{164}$. All VEGF-bound and -unbound receptors can be internalized.

transverse gradients for both uniform and oxygendependent VEGF secretion. ${ }^{32}$ This is due to the parallel nature of the capillaries and the muscle fibers; vascular density changes more quickly in the transverse direction than in the longitudinal direction, and thus so do oxygen and VEGF. We therefore assume that the two-dimensional analysis captures the major features of VEGF distribution.

\section{Blood Flow}

In rats, femoral ligation is used as a model of PAD. Significant, non-uniform collateralization in the hindlimb follows, resulting in large regions of perfused and unperfused muscle. ${ }^{51}$ In addition, average capillary blood velocity is higher in rats that have undergone exercise training. ${ }^{3}$ Thus, using empirical relationships for viscosity and hematocrit distribution, ${ }^{25,46}$ and experimental measurements of blood oxygenation and velocity in ligated rat $\mathrm{EDL}^{3,16,17,44,46}$ we simulated blood flow for perfused tissue and partially perfused tissue (where only half of the tissue arterioles have blood flow).

\section{Tissue Oxygenation}

The distribution of oxygen in the muscle is calculated using our published model of oxygen transport in EDL, ${ }^{25}$ based on the above geometrical and blood flow considerations. In exercise, oxygen consumption increases substantially; for low intensity exercise, we assume here 6-fold over basal (resting) consumption. The blood oxygen saturation in the inlet arterioles $\left(\mathrm{SO}_{2 \mathrm{~A}}\right)$ plummets following ligation, and slowly recovers to the healthy value of $0.6-0.8$ over 2 weeks. ${ }^{23,64}$ Based on measurements of tissue $\mathrm{PO}_{2}$ and hemoglobin saturation calculations ${ }^{16,17,44}$ we estimate $\mathrm{SO}_{2 \mathrm{~A}}$ to be $0.06,0.3$, and 0.6 at 2,7 , and 14 days following ligation, respectively.

\section{Oxygen-dependent VEGF Secretion}

VEGF expression and secretion from each nucleus in the skeletal muscle fibers is dependent on the average oxygen tension $\left(\mathrm{PO}_{2}\right)$ in the myocyte. Although the exact relationship has not been experimentally measured, we used experimental data on the dependence of VEGF release on HIF $1 \alpha$ and $\mathrm{O}_{2}{ }^{26,29,48,59}$ to formulate an empirical relationship between oxygen tension (averaged across the myocyte cross section) and local VEGF release. ${ }^{32}$ Non-exercise trained rats do not increase VEGF secretion in response to hypoxia ${ }^{29,40,47}$ but exercise-trained rats do, ${ }^{29,59}$ and based on experimental data, ${ }^{26,59}$ we assume that the maximum hypoxia-induced secretion increase is six times the level of VEGF secretion in normoxia. ${ }^{24,32}$

\section{VEGF Transport and VEGF-receptor Binding}

VEGF released by the myocyte into the interstitial space diffuses and (in the case of $\mathrm{VEGF}_{164}$ ) can be 
sequestered by binding to GAG chains in the ECM and BM. Reaction-diffusion and mass balance equations are used to describe VEGF transport from the myocyte into the interstitial space and through the ECM and BM. At the endothelial cell surface, VEGF can bind to one or more receptors (Fig. 1). Here we follow the biochemical interaction scheme and kinetics validated in vitro for VEGF isoforms. ${ }^{24,34,36}$ Once bound to cell-surface receptors, VEGF can be internalized by the endothelial cells and degraded.

Concentration of proteins in the ECM is given by an equation of the form:

$$
\partial C_{i} / \partial t=D_{i} \nabla^{2} C_{i}-\sum_{j}^{\text {if } i+j \text { bind }}\left(k_{\mathrm{on}, i, j} C_{i} C_{j}-k_{\mathrm{off}, i j} C_{i j}\right)
$$

where $C_{i}$ and $C_{j}$ are the concentrations of molecules that are present in the interstitial space. Here $C_{i}=\left[\mathrm{V}_{120}\right]$ or $\left[\mathrm{V}_{164}\right] ; C_{j}=[\mathrm{GAG}]$. Note that only $\mathrm{VEGF}_{164}$ (and not $\mathrm{VEGF}_{120}$ ) binds to the GAG chains, so here $C_{i j}=\left[\mathrm{V}_{164} \mathrm{GAG}\right]$ only. The volumetric concentrations, $C$, are expressed in $\mathrm{pM}$, and can be converted to $\mathrm{pmol} /\left(\mathrm{cm}^{3}\right.$ tissue $)$ using geometrical parameters of the tissue (i.e. volume fraction of the interstitial space). Although we have simulated only two isoforms of VEGF, the equations as written allow others to be included.

Concentration of proteins in the BM of either the endothelial cells or the myocytes is given by an equation of the form:

$$
\begin{aligned}
\partial C_{i} / \partial t= & \left(s_{i}+\sum_{m}^{\mathrm{if} i+m \text { bind }}\left(k_{\mathrm{off}, i m} R_{\text {im }}-k_{\mathrm{on}, i, m} C_{i} R_{m}\right)-J_{\mathrm{out}, i}\right) \\
& / d_{\mathrm{BM}}+\sum_{j}^{\mathrm{if} i+j \mathrm{bind}}\left(k_{\mathrm{off}, i j} C_{i j}-k_{\mathrm{on}, i, j} C_{i} C_{j}\right)
\end{aligned}
$$

where $s_{i}$ is the secretion rate of molecule $i$ from the cell; $R_{m}$ is the concentration of receptor $m$ on the cell surface; $R_{i m}$ is the concentration of the cell-surface $i-m$ complex; $J_{\text {out }}$ is the Fickian diffusive flux of VEGF from the $\mathrm{BM}$ to the $\mathrm{ECM}$; and $d_{\mathrm{BM}}$ is the thickness of the basement membrane. In this study, we assume that secretion takes place only from myocytes, and thus $q_{i}=0$ for EBM. Autocrine secretion from endothelial cells can be included by changing this value. In addition, we assume here that endothelial cells express receptors and myocytes do not; therefore the concentration of receptors in the MBM equation is zero. Recent evidence of receptor expression on myocytes could be incorporated simply by making those terms non-zero in the above equation. In this study, $R_{m}$ includes [VEGFR1], [VEGFR2], [NRP1], and [VEGFR1-NRP1]. $R_{i m}$ therefore includes all complexes of those receptors with $\mathrm{VEGF}_{120}$ or $\mathrm{VEGF}_{164}$.
For the receptors and ligand-receptor complexes on the cell surface, equations of the following type are formulated:

$$
\begin{aligned}
\partial R_{m} / \partial t= & s_{m}-k_{\text {int }, \mathrm{m}} R_{m} \\
& -\sum_{i}^{\text {if } i+m \text { bind }}\left(k_{\mathrm{on}, i, m} C_{i} R_{m}-k_{\mathrm{off}, i m} R_{i m}\right) \\
& -\sum_{n}^{\text {if } m+n \text { couple }}\left(k_{\mathrm{couple}, m, n} R_{m} R_{n}-k_{\mathrm{dissoc}, m n} R_{m n}\right)
\end{aligned}
$$

where $s_{m}$ is the insertion rate of newly synthesized receptors into the membrane; $k_{\mathrm{int}, m}$ is the rate of internalization of receptor $m ; k_{\mathrm{couple}, m, n}$ is the kinetic rate of binding of two surface receptors $m$ and $n$ to each other; and $k_{\text {dissoc, } m n}$ is the rate of unbinding of the coupled complex. The cell-surface concentrations of receptors and complexes, $R$, are expressed in units of $\mathrm{mol} / \mathrm{cm}^{2}$, and can be converted to molecules/cell assuming an abluminal endothelial cell-surface area of $1000 \mu \mathrm{m}^{2}$, and to $\mathrm{pmol} /\left(\mathrm{cm}^{3}\right.$ tissue $)$ using the total microvessel surface area per unit volume of tissue.

As receptor tyrosine kinases, dimerization of VEGF receptors is necessary for autophosphorylation and the initiation of intracellular signaling. VEGF receptor dimerization is not explicitly modeled in the formulation presented here, but rather its effects are implicitly included through the kinetics of receptor binding, which are effective rates that incorporate the dimerization step. ${ }^{35}$

Initial VEGF concentrations were obtained from experimental measurements. ${ }^{19}$ The density of VEGF receptors expressed on the surface of endothelial cells in vivo have not yet been measured, however using other experimental measurements we estimated the density in previous studies. ${ }^{24,31,32}$ The receptor densities change for exercise-trained rats and mice following ligation. ${ }^{29,53}$ The VEGF secretion rate is set so that the non-diseased muscle reaches the measured steady state concentration of VEGF, approximately 1 pM..$^{24,31,32,36}$

The above equations are general, and can be used to include additional molecular families that are involved in angiogenesis, for example: PIGF, Neuropilin-2, angiopoietins and Tie receptors, semaphorins and plexins, ephrins and Eph receptors.

\section{Simulations and Solutions}

The blood flow is described by a system of nonlinear algebraic equations (with rheology described by Pries and Secomb ${ }^{46}$ ) that are solved by iterative converging calculation. ${ }^{25}$ The oxygen transport, which depends on the blood flow, is governed by coupled partial differential equations describing intravascular 
and extravascular transport, solved using a numerical finite-difference scheme on an orthogonal $1 \mu \mathrm{m}$ grid. The extravascular equations apply across both the interstitial space and inside the muscle fibers. The VEGF transport equations described above are valid only in the interstitial space and on the cell surface; the muscle fibers and blood vessels are boundary surfaces for the calculations. These were also solved using a uniform orthogonal grid spacing of $1 \mu \mathrm{m}$. At the tissue boundaries, for both oxygen and VEGF transport, periodic conditions were used in the $y$ - and $z$-directions, along with no-flux conditions in the $x$-direction. The steady state solution for each simulation was found using a red-black successive over-relaxation scheme (red-black SOR). ${ }^{13}$ We used a convergence criterion of $10^{-5}$ fractional change per step in $\mathrm{VEGF}_{120}$ and VEGF $_{164}$ at each grid point. The model is coded in $\mathrm{C}++$ and the two-dimensional simulations require approximately $30 \mathrm{~min}$ to reach steady state on a $2.5 \mathrm{GHz}$ Itanium 2 processor with $2 \mathrm{~GB}$ of RAM.

\section{VEGF Concentration and Gradient Measurements}

Both VEGF concentrations and VEGF gradients are thought to be sensed by endothelial cells. ${ }^{15,52}$ Cells do not typically proliferate and migrate simultaneously, and therefore both absolute concentration and relative gradient may play a role in the cellularlevel decision-making process. Both values are difficult or impossible to measure in vivo at the microenvironmental level, and we report predicted values for both in this study.

Absolute VEGF concentrations are reported here as the unbound (free) VEGF concentration in the interstitial space of the tissue. We have previously reported that increased VEGF secretion and decreased VEGF internalization result in increased VEGF concentration. ${ }^{31,36}$ In addition, the presence of Neuropilin can reduce the VEGF concentration by increasing binding to the cell surface. ${ }^{24}$

VEGF gradients are reported in units of \% VEGF/ $10 \mu \mathrm{m}$, and represent the absolute change in VEGF concentration across $10 \mu \mathrm{m}$, divided by the average interstitial VEGF concentration across the cross section of muscle. These are relative gradients, and can thus be converted to absolute gradients by multiplying by the mean interstitial VEGF concentration. Nonuniform VEGF expression (e.g. due to oxygen gradients, or transfection) can result in VEGF gradients, but even for uniform VEGF expression in the tissue, the non-homogenous distribution of the microvessels (and thus the VEGF receptors) results in elevated VEGF interstitial gradients. ${ }^{31}$ We have also shown that increased cell-surface receptor expression can significantly increase the magnitude of VEGF gradients. ${ }^{24,31,32}$ The size of the gradient over $10 \mu \mathrm{m}$ can be compared with the typical length of endothelial tip cells, approximately $30-50 \mu \mathrm{m},{ }^{15,52}$ to determine whether the gradient is steep enough to be sensed by the cell.

\section{RESULTS}

\section{Untreated, Diseased (Femoral-ligated) Muscle}

In rats, the ligation of a femoral artery will result in hindlimb ischemia followed by some recovery due to collateralization. In non-exercise trained rats, however, VEGF secretion is not increased significantly in response to the hypoxia in ischemic parts of the muscle. There is also no change to the VEGF receptor expression. Thus, the VEGF expression remains as for healthy muscle. ${ }^{24}$ VEGF is secreted evenly throughout the muscle, and the VEGF gradients are relatively small, averaging 3.3\%VEGF $/ 10 \mu \mathrm{m}$ (Fig. 2). There is a small variation in the activation levels of the
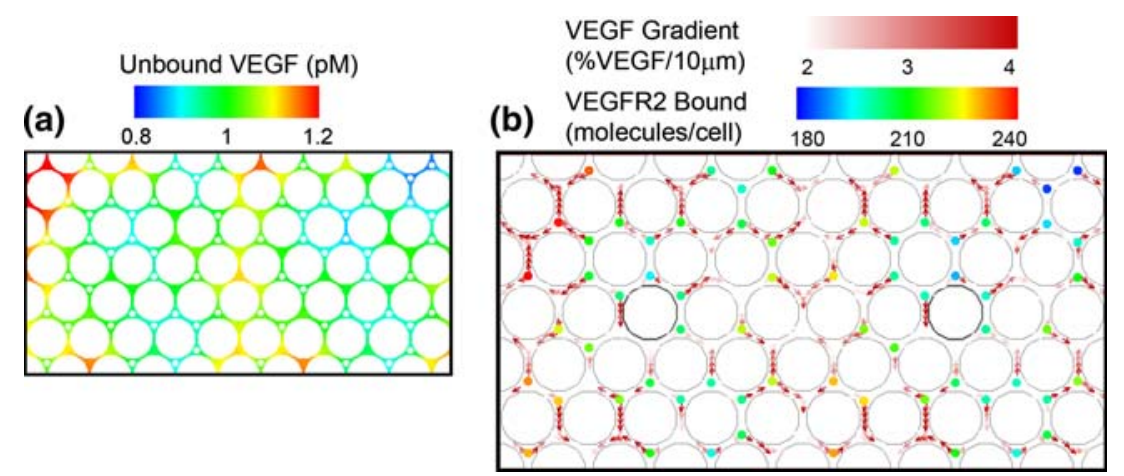

FIGURE 2. VEGF gradients and receptor signaling is limited for untreated ischemic muscle. Simulations of VEGF transport for uniform VEGF secretion from muscle fibers and basal VEGF receptor and NRP1 expression. (a) Distribution of unbound VEGF in interstitial space. (b) VEGF gradients (arrows point in the direction of increasing VEGF concentration) and VEGF-bound VEGFR2 per capillary (236 molecules/cell maximum; 209 molecules/cell average). 
microvessels (with VEGF-VEGFR2 as a surrogate for pro-angiogenic signaling), but not different from healthy tissue. ${ }^{24,31,32}$ This suggests that during ischemia following femoral ligation (as a model of PAD), there is no increase in VEGF signaling, and this may lead to insufficient recovery of tissue perfusion.

Note that even increased (but still uniform) secretion of VEGF results in increased VEGF concentration, but not increased values of the relative VEGF gradients. $^{31}$ Absolute gradients (i.e. the absolute change in concentration of VEGF over a short distance) would increase as the VEGF concentration increases, but it is not yet clear whether endothelial cells respond to relative or absolute gradients.

\section{Cell-based Therapy: VEGF-expressing Myoblasts}

In cell-based pro-angiogenesis therapies, transgenic myoblasts with VEGF secretion rates between 10- and 100-fold basal (0.85-9.3 molecules/myoblast/s for different transgenic clones) are injected into muscle., ${ }^{2,43}$ The myoblasts incorporate into the muscle fibers, and continue to secrete elevated levels of VEGF. However, the spatial heterogeneity of incorporation has not been measured. We simulate myoblast implantation for cases of two myoblasts separated by at least four muscle fibers and of two myoblasts placed adjacent to each other. Each implanted myoblast secretes VEGF at 40-fold above basal level and the size of each myoblast is assumed to be the same as that of a single myonuclear domain (the volume and associated surface are of the multinucleate myocyte under the control of a single nucleus; for rat EDL we have estimated the surface area ${ }^{31}$ at $2500 \mu \mathrm{m}^{2}$ ). Therefore, each implanted myoblast replaces one muscle fiber in our EDL geometry (Fig. 3, stars). VEGF-receptor concentrations (and the VEGF secretion rates of other fibers) remain at basal levels, in this non-exercise-trained rat.

For pro-angiogenic cells separated by at least four muscle fibers (Fig. 3a), maximum VEGF gradients in the interstitial space increase from $12 \% \mathrm{VEGF} / 10 \mu \mathrm{m}$ to $25 \% \mathrm{VEGF} / 10 \mu \mathrm{m}$ and the strongest gradients are directed toward and localized near areas of implanted cells. Maximum VEGFR2 binding on endothelial cells increases from 236 to 740 molecules/cell, three times that of untreated muscle (Fig. 2). For adjacent implanted pro-angiogenic cells, maximum VEGF gradients are increased further to $38 \% \mathrm{VEGF} / 10 \mu \mathrm{m}$ and maximum VEGFR2 binding is increased to 1230 molecules/cell (Fig. 3b). Furthermore, the strongest VEGF gradients are directed toward the implanted cells from all directions in both cases (i.e. VEGF diffuses away from the implanted cells). The synergistic effect of adjacent cell implantation can be further increased if more transgenic cells are placed adjacent to one another. ${ }^{31}$

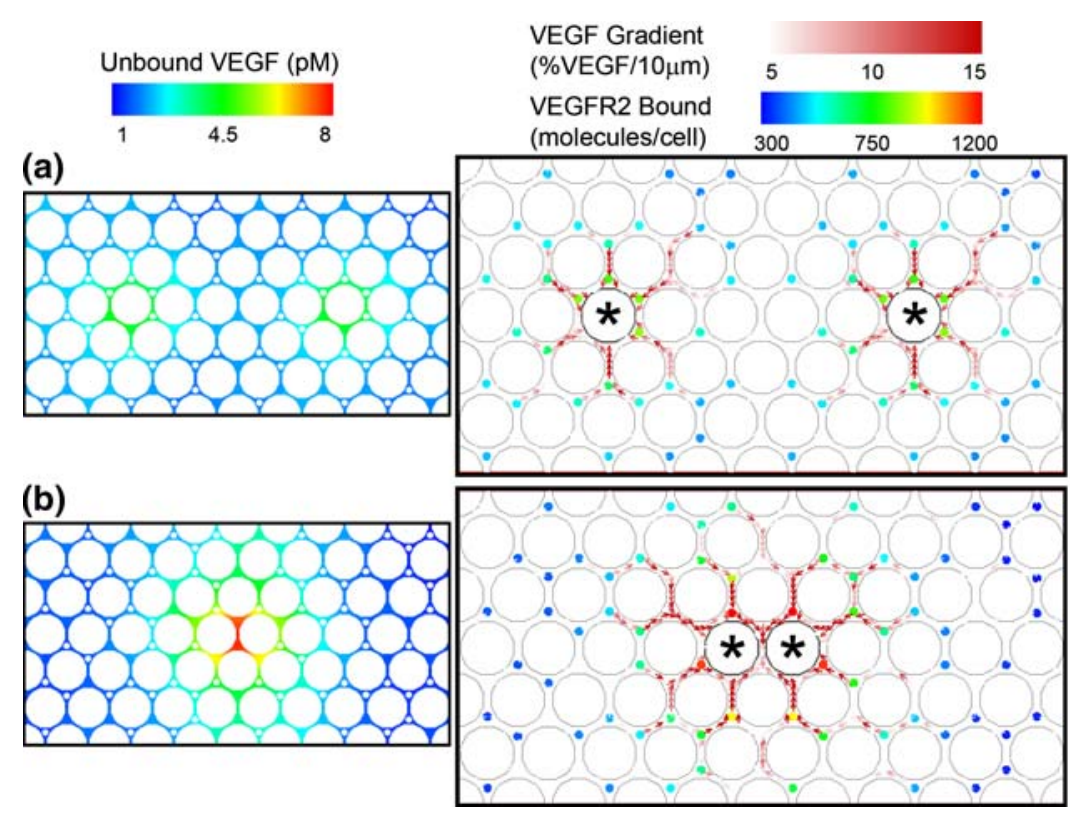

FIGURE 3. Ischemic EDL muscle transplanted with pro-angiogenic cells. Distribution of unbound VEGF in the interstitial space, VEGF gradients (arrows point toward increasing VEGF concentration) and average VEGF-bound VEGFR2 per capillary. Myocytes with $\left(^{*}\right)$ indicate transgenic cells with 40 -fold VEGF secretion rate above basal level. (a) Two pro-angiogenic cells separated by at least four muscle fibers. Maximum VEGF gradient is $25 \%$ VEGF/10 $\mu \mathrm{m}$ and maximum capillary VEGF-bound VEGFR2 is 740 molecules/cell. (b) Adjacent pro-angiogenic cells result in larger gradients (38\%VEGF/10 $\mu \mathrm{m}$ maximum) and increased VEGFR2 binding (1230 molecules/cell maximum). Regardless of spatial placement, the largest VEGF gradients are directed toward proangiogenic cells. 


\section{Gene Therapy: $A A V$-VEGF Construct}

We use AAV-VEGF delivery as an example here, but the results that follow could be applied to many gene delivery strategies for the VEGF pathway, for example, chronic stimulation of HIF to induce VEGF expression, ${ }^{7}$ or use of an engineered zinc-finger binding protein with a transcriptional enhancer to target VEGF regulation. ${ }^{6}$

Experiments for AAV delivery of VEGF gene into skeletal muscle have not quantified VEGF secretion rates per myocyte. In this study, we increase both VEGF $_{120}$ and VEGF $_{164}$ secretion. Transduction efficiency by VEGF AAV inter-arterial delivery has been quantified for rat tibialis anterior (TA) muscle to range between 10 and $20 \% .^{5}$ Also, total VEGF protein content of homogenized TA muscle increases $50 \%$ from local injection of VEGF AAV. ${ }^{4}$ Although the results of the two delivery methods cannot be directly compared, they allow for the estimation of induced VEGF upregulation in affected cells. Total muscle VEGF protein content includes VEGF located within muscle fibers and endothelial cells. However, assuming that a $50 \%$ increase in VEGF protein corresponds to a $50 \%$ increase in total VEGF secretion rate, ${ }^{31}$ and assuming $10 \%$ of myocytes are transduced, this would correspond to a 5-fold increase in VEGF secretion for each affected cell on average. This estimate corresponds to a physiologically relevant VEGF upregulation found in rats during exercise. ${ }^{59}$ Normally, upregulation of VEGF in hypoxia may induce upregulation of VEGF receptors as well, ${ }^{56}$ but hypoxia from PAD does not seem to induce such a response without exercise. Therefore, we assume basal levels of VEGF receptors and Neuropilin.

Staining experiments for AAV transduction in gastrocnemius muscle show that regions of adjacent myocytes become transduced while other regions of adjacent myocytes remain non-transduced. ${ }^{5}$ Therefore, we perform simulations for areas where all skeletal myocytes are transduced, and secrete $\mathrm{VEGF}_{164}$ either uniformly at six times the basal level, or between 3-and 9-times the basal level (randomly per myocyte, averaging six times the basal level for the whole tissue volume) since AAV gene delivery may not be equal for adjacent myocytes (Fig. 4a). We also perform simulations for a region in which all myocytes are transduced (VEGF secretion is six times the basal level) adjacent to a region in which no myocytes are transduced (Fig. 4b).
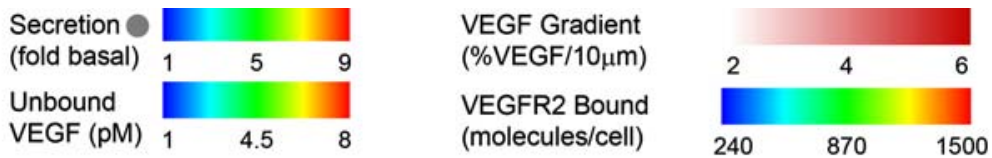

(a)

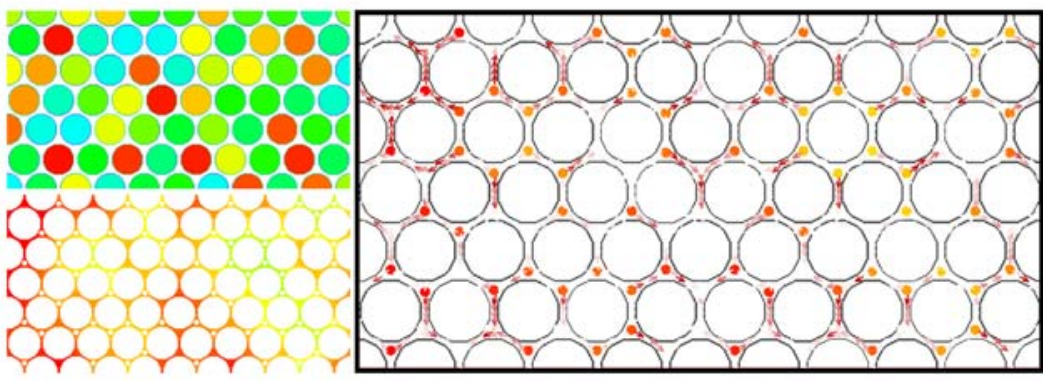

(b)

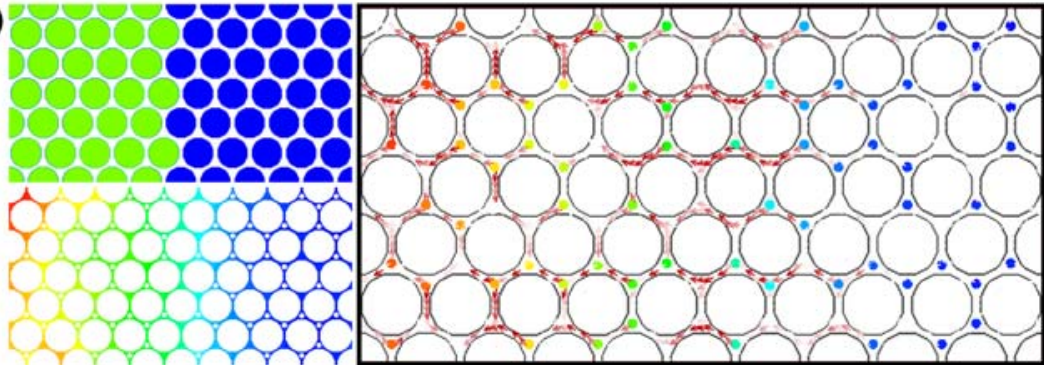

FIGURE 4. Ischemic EDL muscle treated with VEGF $_{164}$ gene delivery. VEGF secretion rate from muscle fibers, distribution of unbound VEGF in the interstitial space, VEGF gradients (arrows point toward increasing VEGF concentration) and average VEGFbound VEGFR2 per capillary. (a) In large regions of transduced muscle fibers, VEGF secretion upregulation (3-9 times basal) increases VEGF gradients (4.1\%VEGF/10 $\mu \mathrm{m}$ average) and VEGFR2 binding (1600 molecules/cell maximum) without preferred orientation. (b) In a region between transduced muscle fibers (VEGF secretion rate six times basal level) and non-transduced fibers, VEGF gradients are directed toward the transduced cells. 
For simulations in a small area where all fibers are transduced, we assume all muscle fibers express VEGF uniformly at six times the basal level. Our results show an average gradient of $3.1 \% \mathrm{VEGF} / 10 \mu \mathrm{m}$ and maximum VEGFR2 binding of 1500 molecules/cell (data not shown). Thus, although VEGF and VEGF-receptor binding have increased, the VEGF gradients have not.

VEGF genes may not be uniformly delivered by AAV. To simulate more heterogeneous delivery, VEGF $_{164}$ secretion from each muscle fiber is randomly chosen between 3- and 9-times the basal level (averaging six times for all fibers in tissue). VEGF gradients increase to $4.1 \% \mathrm{VEGF} / 10 \mu \mathrm{m}$ but VEGFR2 binding is not changed significantly from the uniform 6-fold overexpression (1600 molecules/cell maximum) (Fig. 4a). For VEGF transport between a group of transduced myocytes (each expressing at six times the basal level) and non-transduced myocytes (each expressing at basal level), gradients average $5.8 \% \mathrm{VEGF} / 10 \mu \mathrm{m}$ but can be as high as $27 \% \mathrm{VEGF} /$ $10 \mu \mathrm{m}$ at the boundary between transduced and nontransduced fibers, and gradients are directed towards the region of transduced myocytes (Fig. 4b). Maximum VEGFR2 binding reaches up to 1550 molecules/ cell for endothelial cells located near transduced fibers.

\section{Long-term Exercise Training}

The response of muscle to ischemia is considerably different for exercise-trained rats. Along with a hypoxia-dependent increase in the VEGF secretion, VEGF receptors are upregulated. We have previously shown that the model predicts VEGF signaling to be at its strongest during the first week following femoral ligation. ${ }^{24} \mathrm{We}$ simulate here a time point 7 days after ligation; blood oxygenation has begun to return to normal due to collateralization, and VEGF receptors are upregulated 3.5, 7.2, and 2.1 times control lev$\mathrm{els}^{29,53}$ (VEGFR1, VEGFR2, and Neuropilin, respectively). Although the rat is exercise-trained, the muscle is at rest in this simulation.

For fully perfused tissue (Fig. 5a), we note that although VEGF binding to VEGFR2 is only slightly elevated compared to the non-exercise trained rat, VEGF gradients are considerably higher, averaging $12 \% \mathrm{VEGF} / 10 \mu \mathrm{m}$. VEGF is not upregulated significantly, due to the return of perfusion and thus increased oxygen, but the gradients are higher because of the elevated receptor density. During the exercise period, hypoxia induces VEGF secretion, and both VEGFR activation and VEGF gradients are elevated compared with the untreated case (Table 1).

At the junction between one region of perfused tissue and an unperfused region, the gradients are even higher (Fig. 5b); in addition, hypoxia experienced by the muscle fibers in the non-perfused region results in increased VEGF secretion and increased VEGFVEGFR2 binding.

\section{DISCUSSION}

The stimulation of angiogenesis to improve tissue perfusion in diseases of hypovascularization such as PAD is a fertile area of basic and clinical research. Rat models of PAD (in particular, femoral artery ligation) are helping to answer questions about the pathogenesis and treatment of the disease. In an attempt to further this experimental approach, we have developed a model of rat EDL, in which blood flow, oxygen transport, and VEGF transport and VEGF-receptor activation are combined to predict the impact of various therapies.

The results illustrate the importance of distinguishing between absolute VEGF concentration and relative VEGF concentration gradients. An increase in the absolute concentration of VEGF results in increased VEGFR2 binding and pro-angiogenic signaling. VEGF gradients are important for chemotactic guidance of angiogenic sprouts. It is likely that both increased concentrations and gradients that the cell can sense $\mathrm{e}^{15,52}$ are required for the efficient vascularization of ischemic or hypoxic tissue.

It is possible to change the VEGF concentration without changing the local gradients, and vice versa. For example, a uniform increase in VEGF secretion increases VEGF concentration (and thus VEGFR2 signaling) without a change in gradients. This means, in effect, that all the microvessels experience a similar increase in activation. This can be avoided by altering the spatial distribution of increases in VEGF secretion; either as individual fibers (Fig. 3) or groups of fibers (Fig. 4). The more severe the differences between secretion levels, the steeper the gradients. As a result, certain vessels are more likely to be activated than others (the activation of sprouting from only a subset of vessels is characteristic of angiogenesis).

Similarly, the gradient of VEGF concentration can be increased by elevated receptor expression, without changing the VEGF concentration significantly (Fig. 5a). The action of these two mechanisms together-spatially varying VEGF upregulation and receptor upregulation - may explain why exercise training has been found to be clinically useful in the treatment of PAD, at least for a subset of patients. At the junction between perfused and unperfused regions, high VEGFR2 signaling and high VEGF concentration gradients coincide (Fig. 5b). It would be informative to interrogate whether the responsiveness of certain patients to exercise is a function of their ability to 


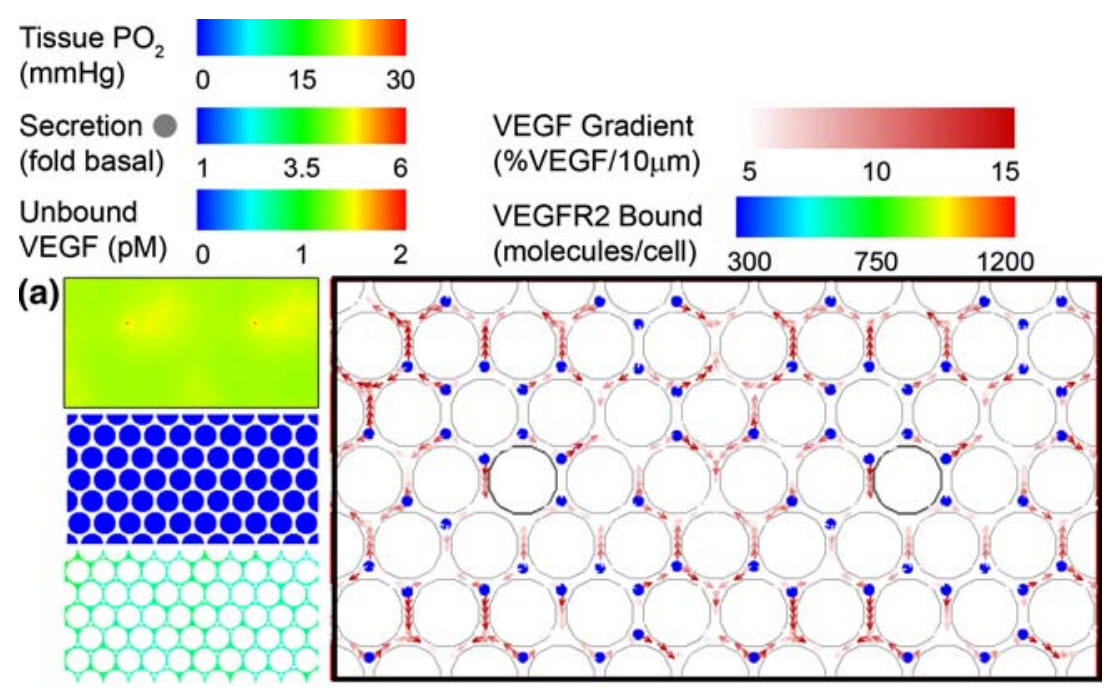

(b)

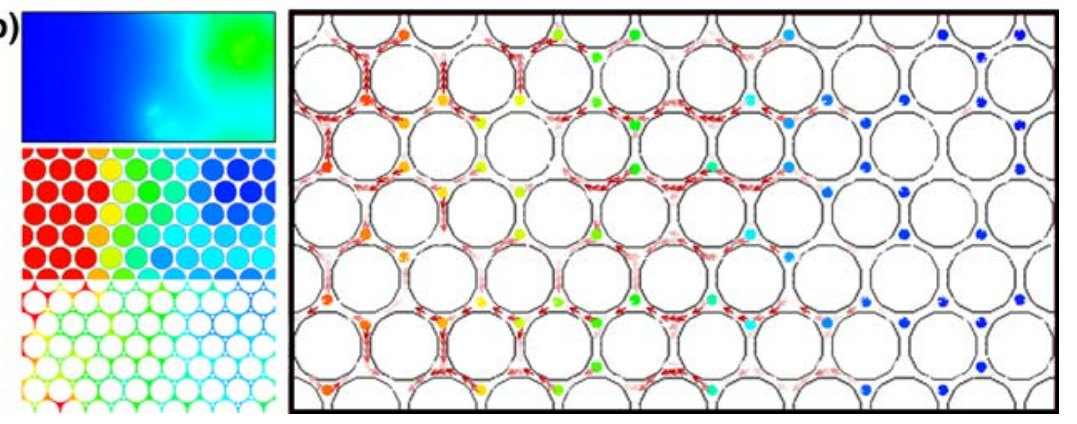

FIGURE 5. Ligated hindlimb EDL muscle in exercise-trained rat. Oxygen distribution, VEGF secretion rate from muscle fibers, distribution of unbound VEGF in the interstitial space, VEGF gradients (arrows point toward increasing VEGF concentration) and average VEGF-bound VEGFR2 per capillary for 1 week after ligation. (a) In fully perfused muscle, VEGF gradients increase (12\%VEGF/10 $\mu \mathrm{m}$ average) vs. untreated muscle, but VEGFR2 binding remains low (332 molecules/cell maximum). (b) In a region between perfused and unperfused tissue, VEGF gradients reach up to $26 \%$ VEGF/10 $\mu \mathrm{m}$ directed towards the unperfused tissue region, and maximum bound VEGFR2 per capillary reaches up to 1670 molecules/cell.

TABLE 1. VEGF gradients and VEGFR2 activation.

\begin{tabular}{|c|c|c|c|c|}
\hline \multirow[b]{2}{*}{ Muscle condition } & \multicolumn{2}{|c|}{$\begin{array}{l}\text { VEGF gradients } \\
(\% \text { VEGF/10 } \mu \mathrm{m})\end{array}$} & \multicolumn{2}{|c|}{$\begin{array}{l}\text { VEGF-VEGFR2 } \\
\text { (molecules/cell) }\end{array}$} \\
\hline & Average & Maximum & Average & Maximum \\
\hline Untreated ligated muscle & 3.3 & 12 & 209 & 236 \\
\hline Cell therapy-distant cells & 3.9 & 25 & 515 & 740 \\
\hline Cell therapy-adjacent cells & 4.1 & 38 & 632 & 1230 \\
\hline Gene therapy-homogenous induction & 3.1 & 12 & 1160 & 1500 \\
\hline Gene therapy-heterogenous induction & 4.1 & 14 & 1160 & 1600 \\
\hline Gene therapy-regional induction & 5.8 & 27 & 1420 & 1550 \\
\hline 7 days exercise training-rest period (fully perfused tissue) & 12 & 23 & 259 & 332 \\
\hline 7 days exercise training—during exercise (fully perfused tissue) & 14 & 28 & 1180 & 1610 \\
\hline 7 days exercise training-rest period (partly perfused tissue) & 13 & 26 & 768 & 1670 \\
\hline 7 days exercise training-during exercise (partly perfused tissue) & 14 & 28 & 1270 & 1790 \\
\hline
\end{tabular}

upregulate VEGF receptor expression, or the robustness of their hypoxia-dependent VEGF secretion.

In summarizing the therapeutic strategies described here, it seems clear that exercise-with concomitant increases in VEGF and VEGF receptor expression-is predicted to be the most effective in inducing angio- genesis. Induction of VEGF alone, either by gene delivery or cell-based methods, does not produce the same level of response. However, it may be possible to combine VEGF delivery with VEGF receptor upregulation; this simultaneous, two-cell approach (i.e. VEGF delivery to, or upregulation in, myocytes; and 
delivery or upregulation of VEGF receptors in endothelial cells) may prove a more widely applicable therapy than exercise.

Although this study is based on the rat EDL muscle, and uses rat experimental data of receptor expression and VEGF secretion, there are clear parallels in human studies. Systems biology approaches such as gene expression microarrays are resulting in the ability to obtain information on the upregulation or downregulation of multiple genes and proteins in healthy, diseased, and treated conditions. For example, in acute skeletal muscle ischemia in humans, VEGF, HIF1 $\alpha$ and VEGFR2 are all upregulated, ${ }^{61}$ along with other pro-angiogenic molecules. However, in chronic muscle ischemia, these molecules are no longer upregulated. ${ }^{61}$ This is in keeping with the rat data, where chronic ischemia due to ligation results in no upregulation of VEGF or its receptors in response to hypoxia. In the rat, exercise training can change this and increase receptor density and VEGF responsiveness to oxygen; one could think of exercise as changing the chronic ischemic response to one of acute ischemia. This may then explain the response of some PAD patients to exercise. It is an open question as to whether a better animal model of ischemic disease will become available.

Systems biology studies such as the one just described are extremely important for the development of models and simulations like those presented here. Human disease is vastly complex and there are many subtleties that it is important to capture in the experimental design; for example, the link and interplay between diabetes and PAD. ${ }^{49}$ To make further progress, we need to combine data-rich biology and physiology with multi-scale computational models of increasing complexity. Additional modules, such as intracellular signaling, ECM proteolysis and tissue remodeling, may be needed for a more complete model of PAD. We hope this study will stimulate further work to characterize the response to diseased states and therapies at the molecular and cellular level.

There are modifications or improvements that can be made in the future to the model and simulations presented here. The muscle geometry (e.g. fiber shape and packing) is somewhat idealized, and while we believe that a representation closer to that observed in explanted tissue will not alter the results significantly, it remains a worthwhile step to take. In addition, we have limited the analysis here to myocyte expression of VEGF and endothelial cell expression of VEGF receptors. In fact, VEGF may be expressed in some amount by the endothelial cells (possibly functioning in an autocrine way), though the experimental evidence is not clear. In addition, myocytes may express VEGF receptors. ${ }^{63,67}$ Both of these points can be incorporated using the equations presented here, but results are not presented here.

The oxygen-HIF $1 \alpha$-VEGF pathway for the determination of oxygen-dependent VEGF secretion was simplified for the purposes of this model. A more detailed model of HIF1 $\alpha$ behavior in response to changing oxygen ${ }^{48}$ may be incorporated to better simulate the oxygen-VEGF link. In vivo experimentation-measurement of microenvironmental oxygen, HIF activation, and VEGF secretion - would also be extremely useful for clarification of this important pathway.

The simulations presented here do not include the resulting changes to the vasculature due to VEGF signaling. Inclusion of such a module would allow the multi-scale model to come 'full-circle': the changes to tissue and vascular geometry can result in re-calculation of the blood flow and oxygen distribution, and thus new inputs for the VEGF transport model. The growth of new blood vessels would bring oxygen to hypoxic tissue and shut down the VEGF upregulation system. We have not yet developed a model of vascular growth to incorporate. Other groups have developed growth models ${ }^{45,58,68}$ though these do not include dependence on VEGF and VEGF-receptor signaling with the detail that our model would require.

Many other protein families besides VEGF, and other molecules such as nitric oxide and nitric oxide synthases (NOS), are involved in angiogenesis, either enhancing or inhibiting neovascularization, and are found to be up- or down-regulated in ischemic disease ${ }^{61}$ Some of these may form part of a therapy for PAD ${ }^{41}$ In addition, the intracellular signaling downstream from VEGF receptors may be altered in the diseased state. The material presented here focuses only on VEGF and its receptors, and therefore we cannot definitively state that angiogenesis is affected only by the parameters of this model. It is a subject of continuing research to determine the relative importance of each of the molecular and cellular processes to pathological, physiological, and therapeutic angiogenesis.

\section{ELECTRONIC SUPPLEMENTARY MATERIAL}

The online version of this article (doi:10.1007/ s10439-007-9303-0) contains supplementary material, which is available to authorized users.

\section{ACKNOWLEDGMENTS}

The authors thank Olga Hudlicka and Margaret D. Brown for valuable discussions of muscle angiogenesis, Brian H. Annex and Christopher D. Kontos for critical 
discussions of peripheral arterial disease, and Peter D. Wagner, Ellen C. Breen, and Kechun Tang for their input on the relationship between HIF1 $\alpha$ and VEGF in skeletal muscle. The study was supported by the National Heart, Lung, and Blood Institute grants HL079653 and HL087351.

\section{REFERENCES}

${ }^{1}$ Annex, B. H., and M. Simons. Growth factor-induced therapeutic angiogenesis in the heart: protein therapy. Cardiovasc. Res. 65:649-655, 2005.

${ }^{2}$ Banfi, A., G. von Degenfeld, and H. M. Blau. Critical role of microenvironmental factors in angiogenesis. Curr. Atheroscler. Rep. 7:227-234, 2005.

${ }^{3}$ Brown, M. D., C. J. Kelsall, M. Milkiewicz, S. Anderson, and O. Hudlicka. A new model of peripheral arterial disease: sustained impairment of nutritive microcirculation and its recovery by chronic electrical stimulation. Microcirculation 12:373-381, 2005.

${ }^{4}$ Byun, J., J. M. Heard, J. E. Huh, S. J. Park, E. A. Jung, J. O. Jeong, H. C. Gwon, and D. K. Kim. Efficient expression of the vascular endothelial growth factor gene in vitro and in vivo, using an adeno-associated virus vector. J. Mol. Cell Cardiol. 33:295-305, 2001.

${ }^{5}$ Chang, D. S., H. Su, G. L. Tang, L. S. Brevetti, R. Sarkar, R. Wang, Y. W. Kan, and L. M. Messina. Adeno-associated viral vector-mediated gene transfer of VEGF normalizes skeletal muscle oxygen tension and induces arteriogenesis in ischemic rat hindlimb. Mol. Ther. 7:44-51, 2003.

${ }^{6}$ Dai, Q., J. Huang, B. Klitzman, C. Dong, P. J. Goldschmidt-Clermont, K. L. March, J. Rokovich, B. Johnstone, E. J. Rebar, S. K. Spratt, C. C. Case, C. D. Kontos, and B. H. Annex. Engineered zinc finger-activating vascular endothelial growth factor transcription factor plasmid DNA induces therapeutic angiogenesis in rabbits with hindlimb ischemia. Circulation 110:2467-2475, 2004.

${ }^{7}$ Date, T., S. Mochizuki, A. J. Belanger, M. Yamakawa, Z. Luo, K. A. Vincent, S. H. Cheng, R. J. Gregory, and C. Jiang. Expression of constitutively stable hybrid hypoxia-inducible factor-1alpha protects cultured rat cardiomyocytes against simulated ischemia-reperfusion injury. Am. J. Physiol. Cell Physiol. 288:C314-C320, 2005.

${ }^{8}$ Deindl, E., I. Buschmann, I. E. Hoefer, T. Podzuweit, K. Boengler, S. Vogel, N. van Royen, B. Fernandez, and W. Schaper. Role of ischemia and of hypoxia-inducible genes in arteriogenesis after femoral artery occlusion in the rabbit. Circ. Res. 89:779-786, 2001.

${ }^{9}$ Desplanches, D., M. H. Mayet, B. Sempore, J. Frutoso, and R. Flandrois. Effect of spontaneous recovery or retraining after hindlimb suspension on aerobic capacity. $J$. Appl. Physiol. 63:1739-1743, 1987.

${ }^{10}$ Dormandy, J. A., and R. B. Rutherford. Management of peripheral arterial disease (PAD). TASC Working Group. TransAtlantic Inter-Society Concensus (TASC). J. Vasc. Surg. 31:S1-S296, 2000.

${ }^{11}$ Ferrara, N. VEGF as a therapeutic target in cancer. Oncology 69(Suppl 3):11-16, 2005.

${ }^{12}$ Ferrara, N., and R. S. Kerbel. Angiogenesis as a therapeutic target. Nature 438:967-974, 2005.
${ }^{13}$ Ferziger, J. H. Numerical Methods for Engineering Application. New York: John Wiley and Sons, 1981.

${ }^{14}$ Flessner, M. F., J. Lofthouse, and R. Zakaria el. In vivo diffusion of immunoglobulin $\mathrm{G}$ in muscle: effects of binding, solute exclusion, and lymphatic removal. Am. J. Physiol. 273:H2783-H2793, 1997.

${ }^{15}$ Gerhardt, H., M. Golding, M. Fruttiger, C. Ruhrberg, A. Lundkvist, A. Abramsson, M. Jeltsch, C. Mitchell, K. Alitalo, D. Shima, and C. Betsholtz. VEGF guides angiogenic sprouting utilizing endothelial tip cell filopodia. J. Cell Biol. 161:1163-1177, 2003.

${ }^{16}$ Heil, M., T. Ziegelhoeffer, S. Wagner, B. Fernandez, A. Helisch, S. Martin, S. Tribulova, W. A. Kuziel, G. Bachmann, and W. Schaper. Collateral artery growth (arteriogenesis) after experimental arterial occlusion is impaired in mice lacking CC-chemokine receptor-2. Circ. Res. 94:671-677, 2004.

${ }^{17}$ Helisch, A., S. Wagner, N. Khan, M. Drinane, S. Wolfram, M. Heil, T. Ziegelhoeffer, U. Brandt, J. D. Pearlman, H. M. Swartz, and W. Schaper. Impact of mouse strain differences in innate hindlimb collateral vasculature. Arterioscler. Thromb. Vasc. Biol. 26:520-526, 2006.

${ }^{18}$ Herzog, S., H. Sager, E. Khmelevski, A. Deylig, and W. D. Ito. Collateral arteries grow from preexisting anastomoses in the rat hindlimb. Am. J. Physiol. Heart Circ. Physiol. 283:H2012-H2020, 2002.

${ }^{19}$ Hoffner, L., J. J. Nielsen, H. Langberg, and Y. Hellsten. Exercise but not prostanoids enhance levels of vascular endothelial growth factor and other proliferative agents in human skeletal muscle interstitium. J. Physiol. 550:217225, 2003.

${ }^{20}$ Hudlicka, O., M. Milkiewicz, M. A. Cotter, and M. D. Brown. Hypoxia and expression of VEGF-A protein in relation to capillary growth in electrically stimulated rat and rabbit skeletal muscles. Exp. Physiol. 87:373-381, 2002.

${ }^{21}$ Hughes, G. C., and B. H. Annex. Angiogenic therapy for coronary artery and peripheral arterial disease. Expert Rev. Cardiovasc. Ther. 3:521-535, 2005.

${ }^{22}$ Idris, N. M., H. Haider, M. W. Goh, and E. K. Sim. Therapeutic angiogenesis for treatment of peripheral vascular disease. Growth Factors 22:269-279, 2004.

${ }^{23}$ Japee, S. A., R. N. Pittman, and C. G. Ellis. Automated method for tracking individual red blood cells within capillaries to compute velocity and oxygen saturation. Microcirculation 12:507-515, 2005.

${ }^{24}$ Ji, J. W., F. Mac Gabhann, and A. S. Popel. Skeletal muscle VEGF gradients in Peripheral Arterial Disease: simulations of rest and exercise, 2006 (submitted).

${ }^{25} \mathrm{Ji}$, J. W., N. M. Tsoukias, D. Goldman, and A. S. Popel. A computational model of oxygen transport in skeletal muscle for sprouting and splitting modes of angiogenesis. $J$. Theor. Biol. 241:94-108, 2006.

${ }^{26}$ Jiang, B. H., G. L. Semenza, C. Bauer, and H. H. Marti. Hypoxia-inducible factor 1 levels vary exponentially over a physiologically relevant range of $\mathrm{O} 2$ tension. Am. J. Physiol. 271:C1172-C1180, 1996.

${ }^{27}$ Kayar, S. R., A. J. Lechner, and N. Banchero. The distribution of diffusion distances in the gastrocnemius muscle of various mammals during maturation. Pflugers Arch. 394:124-129, 1982.

${ }^{28}$ Konopatskaya, O., A. J. Churchill, S. J. Harper, D. O. Bates, and T. A. Gardiner. VEGF165b, an endogenous C-terminal splice variant of VEGF, inhibits retinal neovascularization in mice. Mol. Vis. 12:626-632, 2006. 
${ }^{29}$ Lloyd, P. G., B. M. Prior, H. T. Yang, and R. L. Terjung. Angiogenic growth factor expression in rat skeletal muscle in response to exercise training. Am. J. Physiol. Heart Circ. Physiol. 284:H1668-H1678, 2003.

${ }^{30}$ Lo, A., A. J. Fuglevand, and T. W. Secomb. Oxygen delivery to skeletal muscle fibers: effects of microvascular unit structure and control mechanisms. Am. J. Physiol. Heart Circ. Physiol. 285:H955-H963, 2003.

${ }^{31}$ Mac Gabhann, F., J. W. Ji, and A. S. Popel. Computational model of VEGF spatial distribution in muscle and proangiogenic cell therapy. PLoS Comput. Biol. 2:e127, 2006.

${ }^{32}$ Mac Gabhann, F., J. W. Ji, and A. S. Popel. VEGF gradients, receptor activation, and sprout guidance in resting and exercising skeletal muscle. J. Appl. Physiol. 102:722734, 2007.

${ }^{33}$ Mac Gabhann, F., and A. S. Popel. Model of competitive binding of vascular endothelial growth factor and placental growth factor to VEGF receptors on endothelial cells. Am. J. Physiol. Heart Circ. Physiol. 286:H153-H164, 2004.

${ }^{34}$ Mac Gabhann, F., and A. S. Popel. Differential binding of VEGF isoforms to VEGF receptor 2 in the presence of neuropilin-1: a computational model. Am. J. Physiol. Heart Circ. Physiol. 288:H2851-H2860, 2005.

${ }^{35} \mathrm{Mac}$ Gabhann, F., and A. S. Popel. Dimerization of VEGF receptors and implications for signal transduction: a computational study. Biophys. Chem., in press (2007).

${ }^{36} \mathrm{Mac}$ Gabhann, F., and A. S. Popel. Interactions of VEGF isoforms with VEGFR1, VEGFR2 and Neuropilin in vivo: applications to human skeletal muscle. Am. J. Physiol. Heart Circ. Physiol. 292:H459-H474, 2007.

${ }^{37}$ Maruotti, N., F. P. Cantatore, E. Crivellato, A. Vacca, and D. Ribatti. Angiogenesis in rheumatoid arthritis. Histol. Histopathol. 21:557-566, 2006.

${ }^{38}$ Mathieu-Costello, O., H. Hoppeler, and E. R. Weibel. Capillary tortuosity in skeletal muscles of mammals depends on muscle contraction. J. Appl. Physiol. 66:14361442, 1989.

${ }^{39}$ Milkiewicz, M., M. D. Brown, S. Egginton, and O. Hudlicka. Association between shear stress, angiogenesis, and VEGF in skeletal muscles in vivo. Microcirculation 8:229241, 2001

${ }^{40}$ Milkiewicz, M., O. Hudlicka, J. Verhaeg, S. Egginton, and M. D. Brown. Differential expression of Flk-1 and Flt-1 in rat skeletal muscle in response to chronic ischaemia: favourable effect of muscle activity. Clin. Sci. (Lond.) 105:473-482, 2003.

${ }^{41}$ Milkiewicz, M., E. Ispanovic, J. L. Doyle, and T. L. Haas. Regulators of angiogenesis and strategies for their therapeutic manipulation. Int. J. Biochem. Cell Biol. 38:333-357, 2006.

${ }^{42}$ Osawa, T., M. Onodera, X. Y. Feng, and Y. Nozaka. Comparison of the thickness of basement membranes in various tissues of the rat. J. Electron. Microsc. (Tokyo) 52:435-440, 2003.

${ }^{43}$ Ozawa, C. R., A. Banfi, N. L. Glazer, G. Thurston, M. L. Springer, P. E. Kraft, D. M. McDonald, and H. M. Blau. Microenvironmental VEGF concentration, not total dose, determines a threshold between normal and aberrant angiogenesis. J. Clin. Invest. 113:516-527, 2004.

${ }^{44}$ Paek, R., D. S. Chang, L. S. Brevetti, M. D. Rollins, S. Brady, P. C. Ursell, T. K. Hunt, R. Sarkar, and L. M. Messina. Correlation of a simple direct measurement of muscle $\mathrm{pO}(2)$ to a clinical ischemia index and histology in a rat model of chronic severe hindlimb ischemia. J. Vasc. Surg. 36:172-179, 2002.
${ }^{45}$ Peirce, S. M., E. J. Van Gieson, and T. C. Skalak. Multicellular simulation predicts microvascular patterning and in silico tissue assembly. Faseb J. 18:731-733, 2004.

${ }^{46}$ Pries, A. R., and T. W. Secomb. Microvascular blood viscosity in vivo and the endothelial surface layer. Am. J. Physiol. Heart Circ. Physiol. 289:H2657-H2664, 2005.

${ }^{47}$ Prior, B. M., H. T. Yang, and R. L. Terjung. What makes vessels grow with exercise training?. J. Appl. Physiol. 97:1119-1128, 2004.

${ }^{48}$ Qutub, A. A., and A. S. Popel. A computational model of intracellular oxygen sensing by hypoxia-inducible factor HIF1 $\alpha$. J. Cell Sci. 119:3467-3480, 2006.

${ }^{49}$ Rajagopalan, S., E. Mohler 3rd, R J. Lederman, J. Saucedo, F. O. Mendelsohn, J. Olin, J. Blebea, C. Goldman, J. D. Trachtenberg, M. Pressler, H. Rasmussen, B. H. Annex, and A. T. Hirsch. Regional angiogenesis with vascular endothelial growth factor (VEGF) in peripheral arterial disease: design of the RAVE trial. Am. Heart J. 145:1114-1118, 2003.

${ }^{50}$ Regensteiner, J. G., and W. R. Hiatt. Current medical therapies for patients with peripheral arterial disease: a critical review. Am. J. Med. 112:49-57, 2002.

${ }^{51}$ Rivard, A., M. Silver, D. Chen, M. Kearney, M. Magner, B. Annex, K. Peters, and J. M. Isner. Rescue of diabetesrelated impairment of angiogenesis by intramuscular gene therapy with adeno-VEGF. Am. J. Pathol. 154:355-363, 1999.

${ }^{52}$ Ruhrberg, C., H. Gerhardt, M. Golding, R. Watson, S. Ioannidou, H. Fujisawa, C. Betsholtz, and D. T. Shima. Spatially restricted patterning cues provided by heparinbinding VEGF-A control blood vessel branching morphogenesis. Genes Dev. 16:2684-2698, 2002.

${ }^{53}$ Schiekofer, S., G. Galasso, K. Sato, B. J. Kraus, and $\mathrm{K}$. Walsh. Impaired revascularization in a mouse model of type 2 diabetes is associated with dysregulation of a complex angiogenic-regulatory network. Arterioscler. Thromb. Vasc. Biol. 25:1603-1609, 2005.

${ }^{54}$ Shen, J., R. Samul, R. L. Silva, H. Akiyama, H. Liu, Y. Saishin, S. F. Hackett, S. Zinnen, K. Kossen, K. Fosnaugh, C. Vargeese, A. Gomez, K. Bouhana, R. Aitchison, P. Pavco, and P. A. Campochiaro. Suppression of ocular neovascularization with siRNA targeting VEGF receptor 1. Gene Ther. 13:225-234, 2006.

${ }^{55}$ Shibuya, M., and L. Claesson-Welsh. Signal transduction by VEGF receptors in regulation of angiogenesis and lymphangiogenesis. Exp. Cell Res. 312:549-560, 2006.

${ }^{56}$ Shweiki, D., A. Itin, D. Soffer, and E. Keshet. Vascular endothelial growth factor induced by hypoxia may mediate hypoxia-initiated angiogenesis. Nature 359:843-845, 1992.

${ }^{57}$ Soker, S., H. Q. Miao, M. Nomi, S. Takashima, and M. Klagsbrun. VEGF165 mediates formation of complexes containing VEGFR-2 and neuropilin-1 that enhance VEGF165-receptor binding. J. Cell Biochem. 85:357-368, 2002.

${ }^{58}$ Sun, S., M. F. Wheeler, M. Obeyesekere, and C. W. Patrick Jr. A deterministic model of growth factor-induced angiogenesis. Bull. Math. Biol. 67:313-337, 2005.

${ }^{59}$ Tang, K., E. C. Breen, H. Wagner, T. D. Brutsaert, M. Gassmann, and P. D. Wagner. HIF and VEGF relationships in response to hypoxia and sciatic nerve stimulation in rat gastrocnemius. Respir. Physiol. Neurobiol. 144:71-80, 2004.

${ }^{60}$ Trentin, D., H. Hall, S. Wechsler, and J. A. Hubbell. Peptide-matrix-mediated gene transfer of an oxygeninsensitive hypoxia-inducible factor-1alpha variant for 
local induction of angiogenesis. Proc. Natl. Acad. Sci. USA 103:2506-2511, 2006.

${ }^{61}$ Tuomisto, T. T., T. T. Rissanen, I. Vajanto, A. Korkeela, J. Rutanen, and S. Yla-Herttuala. HIF-VEGF-VEGFR2, TNF-alpha and IGF pathways are upregulated in critical human skeletal muscle ischemia as studied with DNA array. Atherosclerosis 174:111-120, 2004.

${ }^{62}$ Tyml, K., O. Mathieu-Costello, L. Cheng, and E. G. Noble. Differential microvascular response to disuse in rat hindlimb skeletal muscles. J. Appl. Physiol. 87:1496-1505, 1999.

${ }^{63}$ van Weel, V., M. M. Deckers, J. M. Grimbergen, K. J. van Leuven, J. H. Lardenoye, R. O. Schlingemann, G. P. van Nieuw Amerongen, J. H. van Bockel, V. W. van Hinsbergh, and P. H. Quax. Vascular endothelial growth factor overexpression in ischemic skeletal muscle enhances myoglobin expression in vivo. Circ. Res. 95:58-66, 2004.

${ }^{64}$ Ward, K. R., I. Torres Filho, R. W. Barbee, L. Torres, M. H. Tiba, P. S. Reynolds, R. N. Pittman, R. R. Ivatury, and
J. Terner. Resonance Raman spectroscopy: a new technology for tissue oxygenation monitoring. Crit. Care Med. 34:792-799, 2006.

${ }^{65}$ Waters, R. E., R. L. Terjung, K. G. Peters, and B. H. Annex. Preclinical models of human peripheral arterial occlusive disease: implications for investigation of therapeutic agents. J. Appl. Physiol. 97:773-780, 2004.

${ }^{66}$ Whitaker, G. B., B. J. Limberg, and J. S. Rosenbaum. Vascular endothelial growth factor receptor-2 and neuropilin-1 form a receptor complex that is responsible for the differential signaling potency of $\operatorname{VEGF}(165)$ and VEGF(121). J. Biol. Chem. 276:25520-25531, 2001.

${ }^{67}$ Williams, R. S., and B. H. Annex. Plasticity of myocytes and capillaries: a possible coordinating role for VEGF. Circ. Res. 95:7-8, 2004.

${ }^{68}$ Zheng, X., S. M. Wise, and V. Cristini. Nonlinear simulation of tumor necrosis, neo-vascularization and tissue invasion via an adaptive finite-element/level-set method. Bull. Math. Biol. 67:211-259, 2005. 